\title{
Efficient production of active form of recombinant cassava hydroxynitrile lyase using Escherichia coli in low-temperature culture
}

\author{
Hisashi Semba • Eita Ichige • Tadayuki Imanaka •
} Haruyuki Atomi $\cdot$ Hideki Aoyagi

Published online: 19 July 2008

(C) Springer-Verlag 2008

\section{Erratum to: Appl Microbiol Biotechnol}

\section{DOI 10.1007/s00253-008-1464-8}

The original version of this article unfortunately contained a mistake. The positions of the asterisks of Fig. 1 were shifted. The correct version is given here. 
Fig. 1 Codon substitution of MeHNL gene. Upper - codon substituted MeHNL sequence (modSHNL-3). Lower - natural MeHNL sequence (GeneBank accession no. Z29091). Identical base is indicated by an asterisk

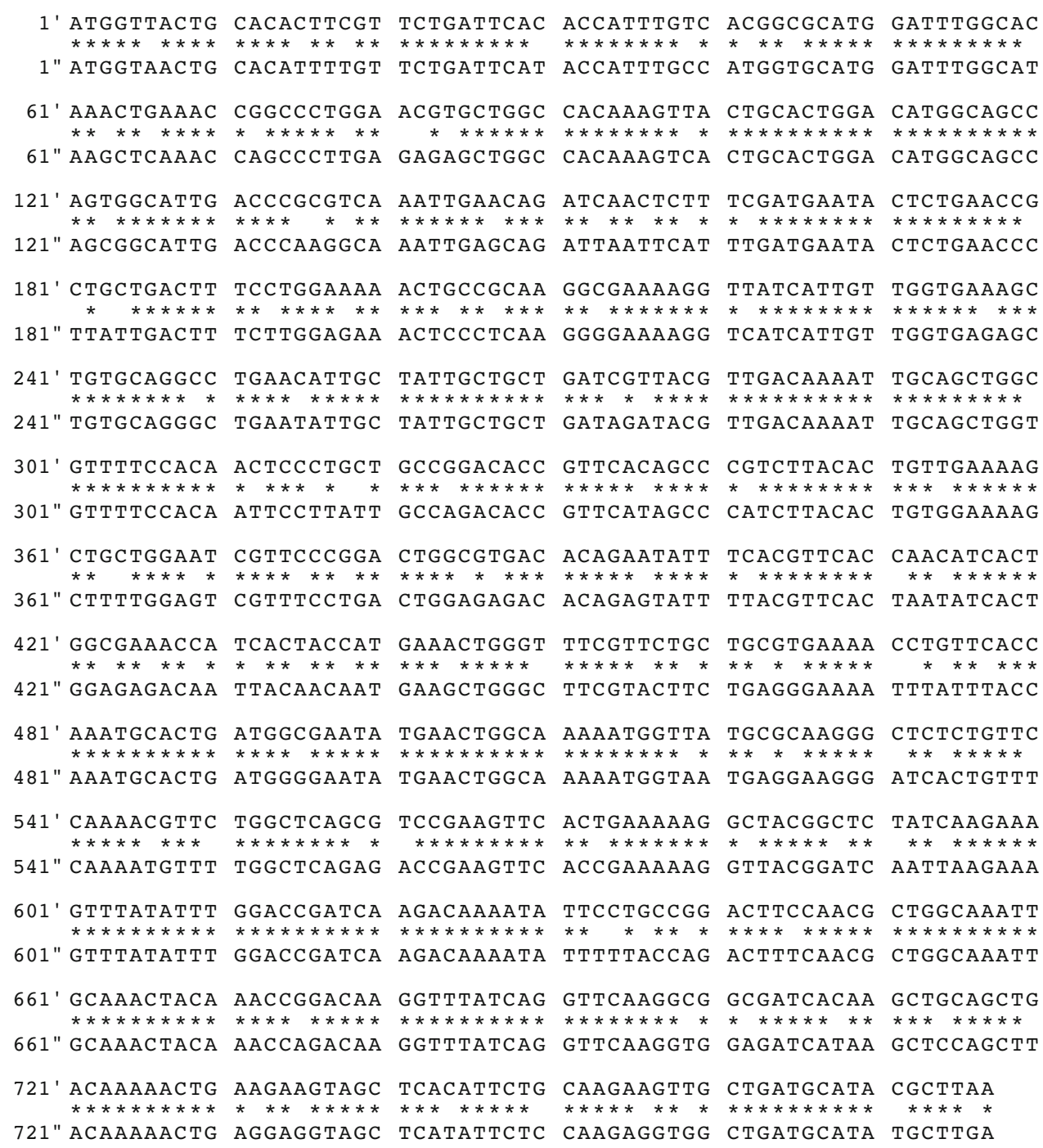

